\title{
Electron Density Distribution and Particle Precipitation in South Atlantic Anomaly: Report on Gyro-Plasma Probe Observation Installed on TAIYO
}

\author{
Hiroshi Oya, Tadatoshi TAKahashi, and Akira Morioka \\ Geophysical Institute, Tohoku University, Sendai, Japan
}

(Received September 5, 1978)

\begin{abstract}
Analyses have been made on the electron density and ion sheath capacity data obtained by gyro-plasma probe installed on TAIYO. The data for 160 orbits provide various states of the electron density profile in the ionosphere, such as the local time dependence and the equatorial anomaly. The measured impedance values at $406 \mathrm{kHz}$ reveals the ion sheath thickness surrounding the probe. In the region of the South Atlantic anomaly of the geomagnetic field, quick changes of the ion sheath capacitance have been observed indicating charging up of the probe. These quick changes are separated into two types; the first is the quick sharp variation and the second is the gradual variation. The sharp variation of the sheath capacitance value is disclosed to be triggered by the telemetry radio wave in the region of the South Atlantic anomaly.
\end{abstract}

\section{Introduction}

Observation of the electron number density in low latitude ionosphere have been carried out by a gyro-plasma probe (a swept frequency impedance probeIMP) installed on TAIYO, third scientific observation satellite in Japan, that was launched on 24th, February 1975, into an orbit with an inclination of $-31.5^{\circ}$ and with the initial perigee of $255 \mathrm{~km}$ and the initial apogee of 3,136 $\mathrm{km}$. After the launch TAIYO had transmitted the data during about 2 years. During this period the IMP observation was made for 160 cases of full orbit observations because the capability of power supply from the solar cell is limited. The gyro-plasma probe had been developed originally as a kind of swept frequency impedance probe that has a system of special performance to eliminate the disturbance effect of the stray capacitance (OYA and OBAYASHI, 1966). The swept frequency impedance probe was installed on the satellite with additional function to arrange the observed data for the transmission through the PCMtelemetry system with the low bit rate of $64 \mathrm{bits} / \mathrm{sec}$ (EJIRI et al., 1973; OYA and MORIOKA, 1975). In the system of IMP, the upper hybrid resonance frequency and the capacitance value of the spherical probe (radius $6 \mathrm{~cm}$ ) at 406 
$\mathrm{kHz}$ are measured (OYA and MoRIOKA, 1975). The purpose of the present paper is to give the results of the analyses of IMP data on the electron density obtained from the measured upper hybrid resonance frequency and the charging up effects of the satellite and probe deduced from the sheath capacitance.

\section{Data Processing}

The instrumentation of the gyro-plasma probe (IMP) installed on TAIYO has already been described in detail (OYA and MORIOKA, 1975) we will not give description here. The original data format of the satellite TAIYO is composed of the frame that consists of 32 words. The basic clock is set to send one frame in $4 \mathrm{sec}$ in real-time mode operation. The three words are used as i) the capacitance value of the probe at $406 \mathrm{kHz}$, ii) status of the low-pass filter and iii) frequency of the upper hybrid resonance. Since automatic detection system has been employed to measure the upper hybrid resonance, the detection of the resonance point is largely affected by low frequency noise on the probe; this noise is mainly comes from the inside the satellite, through the solar cells that contact to space plasma, that has clock to control PCM data. To eliminate this noise effects we used two types of low pass filters. The two filters are switched every $4 \mathrm{sec}$ (corresponding to the switching of the telemetry frame), when the cut off frequency $40 \mathrm{~Hz}$ is used, the operation is called L-mode and that is called $\mathrm{H}$-mode when the other filter with cut off frequency $150 \mathrm{~Hz}$ is used. The data of about 160 full path coverages are processed by digital computer to calculate the electron density, and the capacitance of the probe, together with the orbital parameter to give satellite position, and geomagnetic field strength. The observation results are summarized in forms of the data tables and data plots for every revolutions. The operation of L-mode indicates an effective improvement of the automatic detection of the UHR (upper hybrid resonance): the observed data however still contain certain rate of miss detection due to noise. Figure 1 shows an example of the observed $f_{\mathrm{UHR}}$ for the observation in revolution 23; open circles indicate the observed data for the high mode and the solid circles indicate the low mode of the low-pass filter, respectively. In the data of Fig. 1, three trends, marked with A, B and C, are noted. The trend A, observed near the frequency of $11.0 \mathrm{MHz}$, can be attributed to instrumental cause under a very noisy condition; the automatic detection is started for the operation at the time just after the feeding of signal. Almost all data that belong to this trend is observed in the case of the high mode operation. The trend B, that can be traced middle in the figure, is the detection near the upper hybrid resonance but in the higher part of the frequency. The most possible cause of this detection is the existence of the Bernstein mode resonance near at the $n f_{c}$, where $n$ 


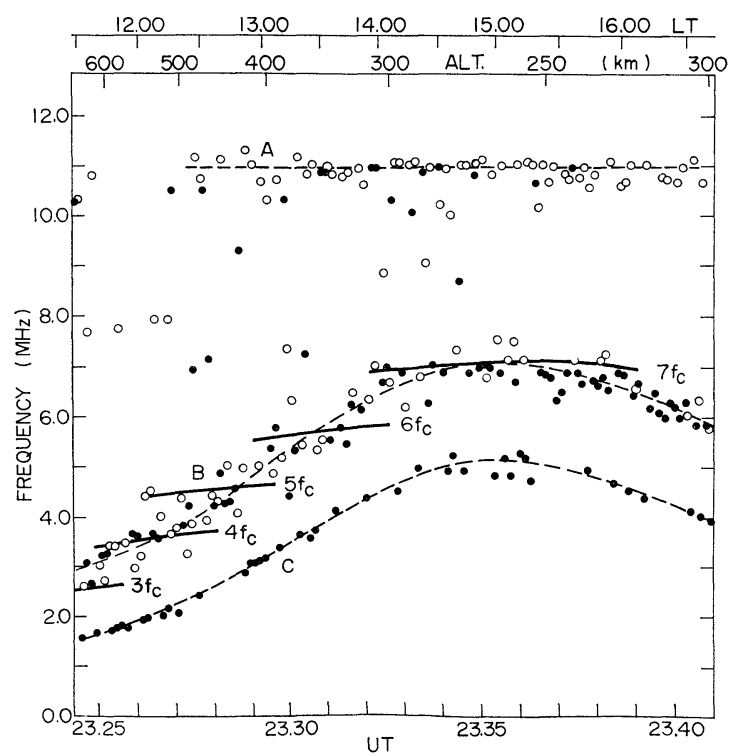

Fig. 1. The W16 data indicated by the frequency versus time or satellite position; open circles indicate the data observed at the high mode of the noise filter and solid dots indicate the data observed at the low mode of the noise filter. For comparison, frequencies of $n f \mathrm{c}$ are indicated with short solid curves.

is the integer to indicate the trend of the harmonic number. It can be noted that the trend of $n f_{\mathrm{c}}$ is observed almost in parallel to $f_{\mathrm{UHR}}$. This is caused by the characteristics of the automatic detection system, i.e., we can detect the $n f_{\mathrm{c}}$ resonance only when the level of the impedance value becomes lower than a given threshold level. This threshold level is set to discriminate the sheath resonance from the upper hybrid resonance frequency that reveals a remarkable dip. The detection of $n f_{\mathrm{c}}$ is therefore limited around the upper hybrid resonance. A logic system is also installed in this automatic resonance detection system; once a resonance point has been detected in a given sweep interval of the frequency applied to the probe, the operation of the automatic detection is stopped and the searching process is reset from the high frequency side of the sweep range. Therefore the detection of $n f_{\mathrm{c}}$ is made in a range $f_{\mathrm{s}}>n f_{\mathrm{c}}>f_{\mathrm{UHR}}$, where $f_{\mathrm{s}}$ is the frequency where the impedance value coincides with the threshold level. The upper hybrid resonance is characterized by a large dip that can still be detectable using the filter with the cut off frequency of $40 \mathrm{~Hz}$, i.e., low-mode of the low-pass filter. The trend $C$ in the figure is, then, indicating correct UHR resonance frequency. The results of the automatic resonance detection become ineffective when the resonance frequency approaches to the 
upper limit $11.0 \mathrm{MHz}$; in this case, however, we can use the capacitance value at $406 \mathrm{kHz}$ to extrapolate the estimation of the electron number density. The spherical probe surrounded by the ion sheath in the ionospheric height, has a capacitance of the concentric spherical condenser as

$$
C=\frac{4 \pi \varepsilon_{0}\left(r_{0}^{2}+r_{0} l\right)}{l}
$$

where $r_{0}$ is radius of the spherical probe, and $l$ is the sheath thickness. The sheath thickness $l$ is comparable to the Debye's shielding length $\lambda_{\mathrm{D}}$ and expressed introducing a correction factor $\xi$, as

$$
l=\xi \cdot \lambda_{\mathrm{D}}=\xi \cdot \sqrt{\frac{\varepsilon_{0} \kappa T_{\mathrm{e}}}{e^{2} N}}
$$

where $T_{\mathrm{e}}$ and $\kappa$ are the electron temperature and Boltzmann constant, respectively. Substituting Eq. (2) into Eq. (1) it follows that

$$
C=4 \pi \varepsilon_{0} r_{0}+\frac{4 \pi \varepsilon_{0} r_{0}^{2} \cdot e}{\xi \sqrt{\varepsilon_{0} \kappa T_{e}}} \cdot \sqrt{N}
$$

i.e., the capacitance indicates a linear relationship to the square root of electron number density $N$. Figure 2 shows the relation between the electron number density calculated from $f_{\mathrm{UHR}}$ and capacitance value measured at $406 \mathrm{kHz}$, for several satellite paths. Clearly defined linear relations are observed in the figure in which the abscissa and the ordinate represent the square root of electron number density and capacitance at $406 \mathrm{kHz}$, respectively; i.e., this indicates

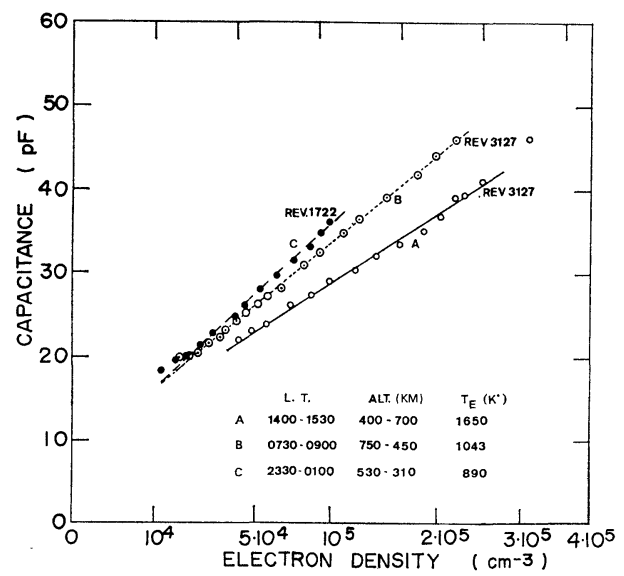

Fig. 2. The measured sheath capacity $C$ versus $\sqrt{N}$ where $N$ is the electron density; the linear relation indicates that the ionospheric electron temperature is nearly constant in a given region. 
that the electron temperature is nearly constant within an indicated range, and the gradient of the line contains an information on the electron temperature of the ionosphere. The electron temperature is obtained assuming $\xi=2.5$ as indicated in the figure with the local time and the altitude ranges. Assuming a constant electron temperature near $F$-layer peak along the satellite path the number density can thus be determined from observed sheath capacitance, even the $f_{\mathrm{UHR}}$ frequency approaches to the detection limit. When a quick variation of the electron temperature takes place, this results an error for evaluated number density that corresponds to the resonance frequency in a range higher than $11.0 \mathrm{MHz}$. When the number density becomes smaller than $5 \times 10^{4} / \mathrm{cm}^{3}$ the observed points tend to leave from straight lines as shown in the cases of $B$ and $\mathrm{C}$ in the figure. In this case more detailed treatment with thick sheath model is required to calculate the relation between the electron number density and the capacitance, as will be discussed in Section 4 .

\section{Results on Electron Density Distribution}

\subsection{Typical observation results in daytime ionosphere}

An example of data plot for revolution 2996 is shown in Fig. 3; the observation at ionospheric height was carried out in daytime for this revolution as has been given at the bottom of the figure. The electron number density above about $10^{6}$ electrons $/ \mathrm{cm}^{3}$ is hardly determined by the automatic resonance detection limit mentioned in the previous section. The extrapolation using the sheath capacity value can be used for the determination of the electron density for this case near at $F$-layer peak. The deduced electron number densities of the $F$-layer peaks are $8.1 \times 10^{5} / \mathrm{cm}^{3}$ at $9: 30 \mathrm{LT}$ and altitude of $308 \mathrm{~km}$ and $9.1 \times$ $10^{5} / \mathrm{cm}^{3}$ at $12: 00 \mathrm{LT}$ and $320 \mathrm{~km}$, respectively. Equatorial anomaly of the electron density distribution is one of the important subjects of TAIYO. One of density minima in Figs. 3 and 4 coincides all with perigee of the satellite orbit but the other of the minima takes place very close to the geomagnetic equator (see arrows in the figures). So the density maxima are at least a mixture of crossing two times the $F_{2}$-layer and the equatorial anomaly. One of dips that reveals near at the geomagnetic equator should take place at the magnetic equator defined by zero inclination.

\subsection{Typical observation results in night time ionosphere}

In Figs. 5 and 6 typical results for the observation in nighttime ionosphere are represented. The local times of subsatellite point at perigee are $02: 20 \mathrm{LT}$ and $03: 05$ LT for revolution 1722 and 1782, respectively. The observed peak of electron number densities of the $F$-layer are $1.1 \times 10^{5}$ electrons $/ \mathrm{cm}^{3}$ at local 


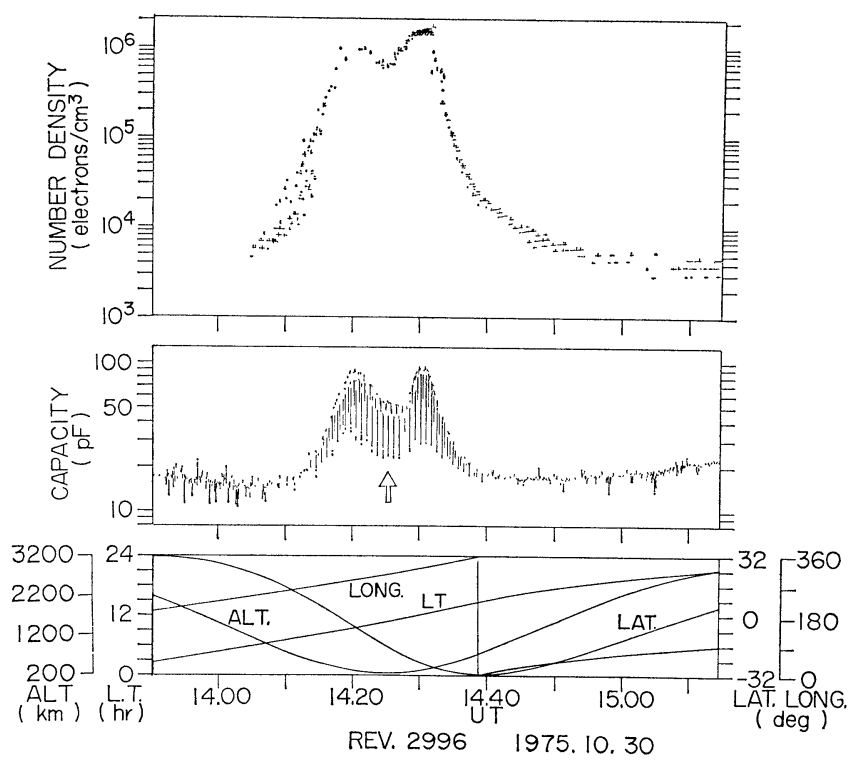

Fig. 3. An example of the data during one path of TAIYO observed in the revolution number 2996 on Oct. 30, 1975; the electron density and the sheath capacity are indicated versus UT: the ionosphere was observed during daytime in this case. The satellite position in terms of latitude (LAT.), longitude (LONG.), local time (LT) and altitude (ALT.) are given as the function of UT. The satellite crosses the geomagnetic equator at position given by an arrow.

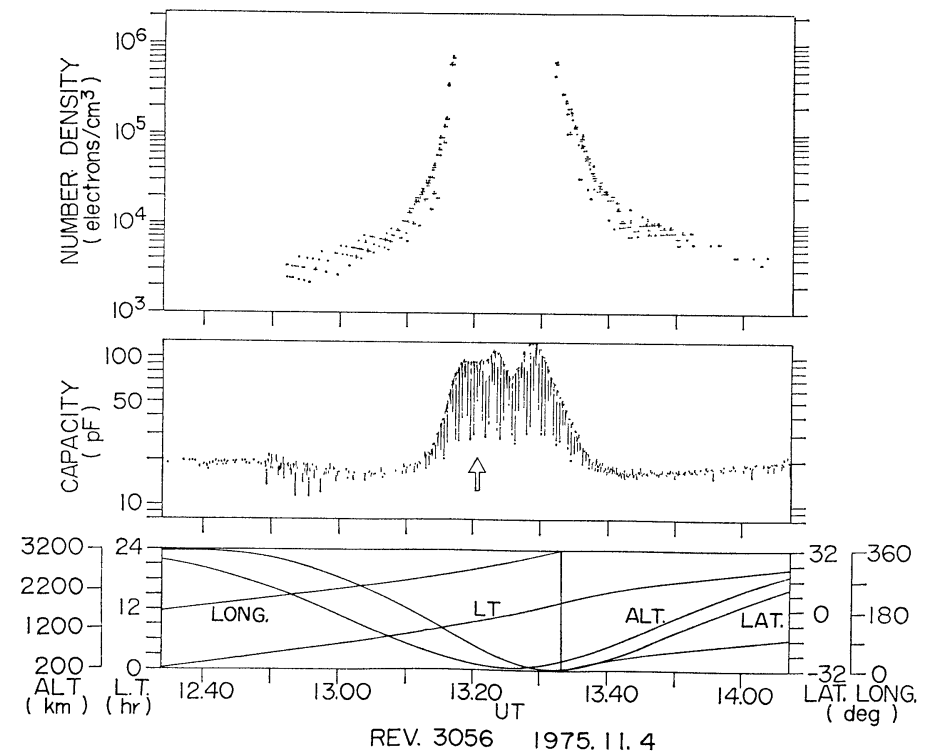

Fig. 4. Same as Fig. 3, for the data obtained during the revolution 3056 on Nov. 4, 1975. 


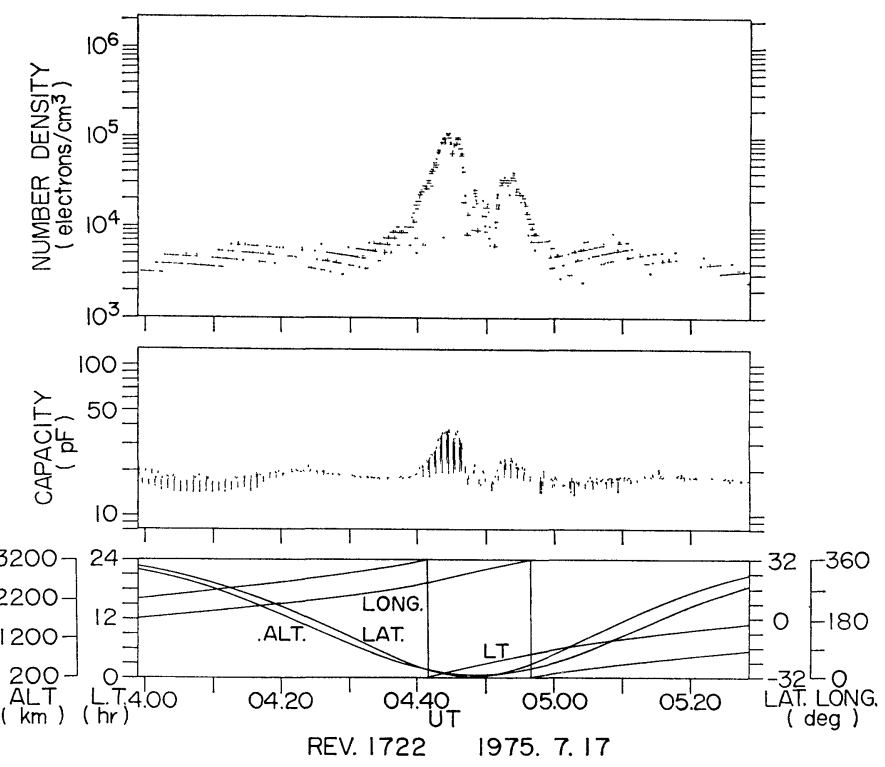

Fig. 5. Same as Fig. 3, for the nighttime ionosphere obtained during the revolution 1722 on July 17, 1975.
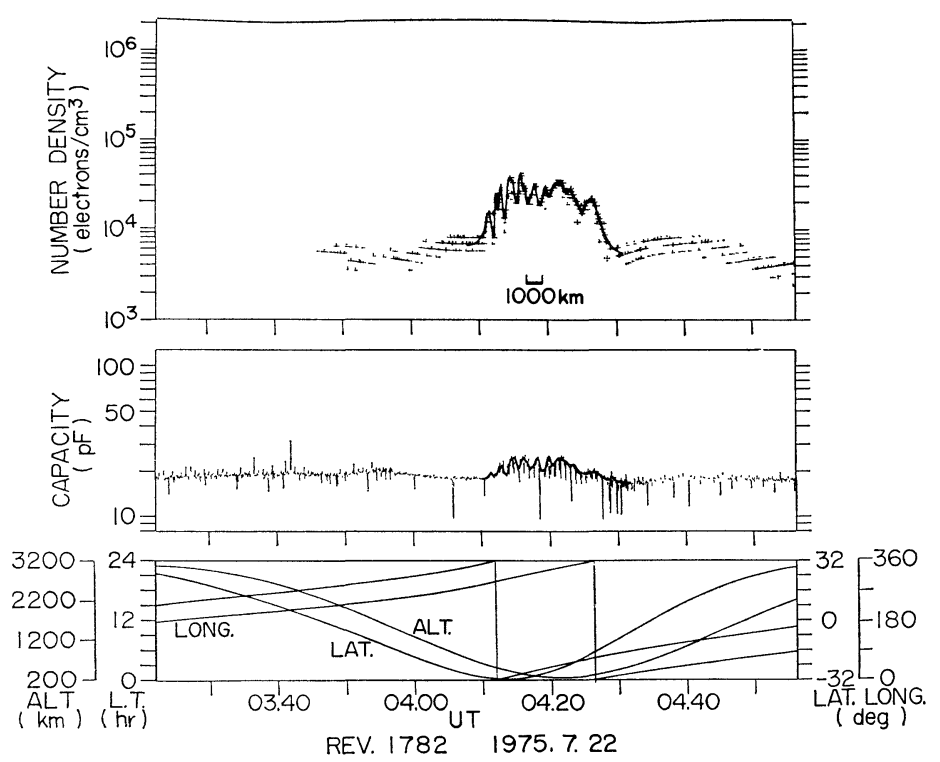

Fig. 6. Same as Fig. 3, for the nighttime ionosphere obtained during the revolution 1782 on July $22,1975$. 
time $00: 55 \mathrm{LT}$ and altitude of $315 \mathrm{~km}$ and $4.0 \times 10^{4}$ electrons $/ \mathrm{cm}^{3}$ at $04: 05 \mathrm{LT}$ and $335 \mathrm{~km}$ for revolution 1722 . The number density indicates low value as $1.35 \times 10^{4}$ electrons $/ \mathrm{cm}^{3}$ around the perigee, corresponding to night time decay of the electron density in the bottom side ionosphere. One of the newly observed results of the nighttime ionosphere are irregular substructures of the electron density distribution. As has been indicated in figures, fluctuatious structure are observed both in the profiles of the electron number density and the sheath capacity; and a good correlation between them is evident. In the profile of the sheath capacity very sharp change of capacity is seen clearly. From the accurate periodicity of this variation, it is concluded that the structure is due to crossing of the probe through the satellite wake. We should then distinguish this wake effect from the natural fluctuation mentioned above.

\subsection{Local time dependence of the ionospheric structure}

Examples of the ionospheric height distribution are shown in Fig. 7, for the observation of different local time. In the diagram, local times at observing position are indicated with bars in the figure. In revolution 3187 , the profile indicated by broken line is obtained by extrapolation using observed sheath capacity. The cases of revolutions 2805 and 3187 indicate that the ionosphere

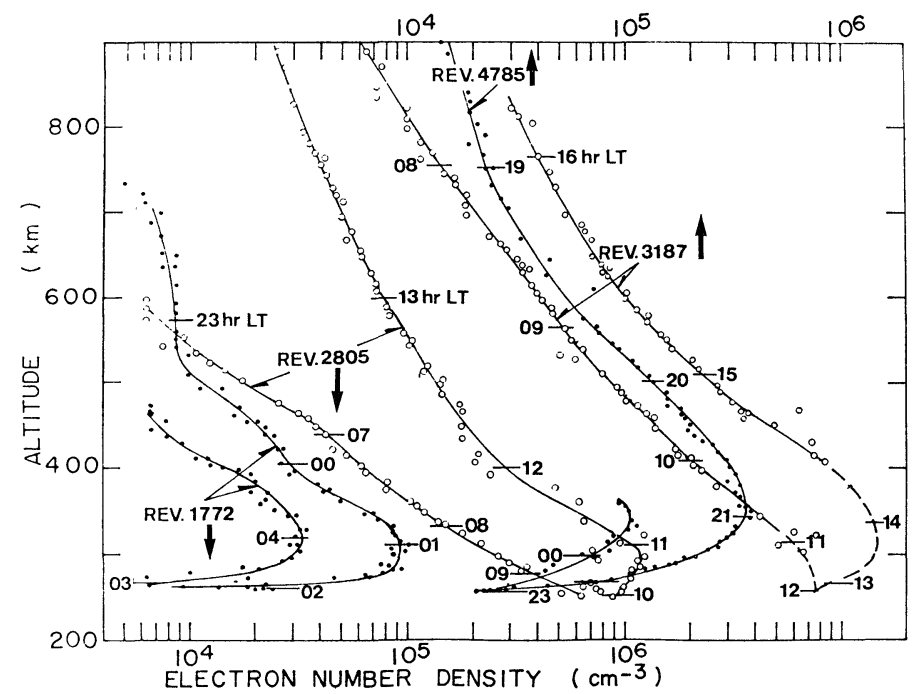

Fig. 7. Examples of the height distribution of the electron density for different local time; since each curves represent the observation during 3 or $4 \mathrm{hr}$, as indicated in the diagram, the height distribution include a modulation by local time. The scale for the electron density values should be read at the corresponding side (top or bottom) as indicated by arrows. 
grows during morning to afternoon while the decay of the ionosphere from evening to midnight is seen in revolution 4785 . The ionosphere continues to decay from midnight to predown as shown for the case of revolution 1722 . Though the distribution modified by a component of the local time passage, the electron density profile versus altitude reflects the ionospheric scale height of the ion distribution. Sometimes the transition height, at which the step wise change of the scale height is observed, can be identified from the profiles. Obtained transition heights are about 540 and $700 \mathrm{~km}$ for the cases of revolutions 1722 and 4785, respectively. These results are consistent with the results of ion composition detected by RPA installed on TAIYO (Miyazaki, private communication, 1977). Local time dependence of the peak density of the $F$-layer and density at $1,350 \mathrm{~km}$ altitude is shown in Fig. 8. For comparison the results obtained by the satellite ISIS-2 topside sounder received at low latitude station (Santiago) in the same period are also plotted with crosses in the figure; i.e., data of ISIS-2 from July to November, 1975 are selected so as to coincide with the period included in the observational periods of IMP installed on TAIYO.

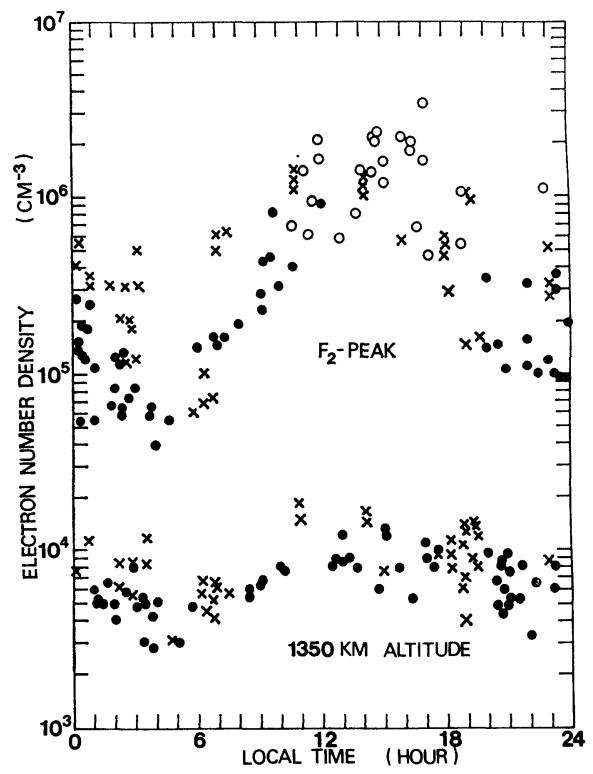

Fig. 8. Local time dependence of the electron density, at the maximum of the $F_{2}$-layer $\left(F_{2}\right.$-PEAK $)$ and at the level of $1,350 \mathrm{~km}$, observed during July 1 to November 30, 1975. The solid circles (Open circles are used when the data are deduced from the sheath capacity) indicate the data obtained by TAIYO; and crosses indicate the data obtained by ISIS. 
Though, in both cases of the observations, the peak densities are distributed with rather scattered nature, principal trend of the results of the two satellites show good agreement for the dependence on the local time and average values. The local time dependence of the topside ionosphere at $1,350 \mathrm{~km}$ level also shows reasonable coincidence.

\section{Charging Effects}

\subsection{Sheath effects}

The sheath capacity measured at $406 \mathrm{kHz}$ provides informations on the ion sheath thickness surrounding the probe. The formation of the ion sheath is an essential evidence associated with the boundary of the material and plasma; many works have been carried out to describe the sheath structure (OYA, 1965; BuCKLEY, 1966, 1967; Aso, 1973; KIST, 1977) and effects on the radio frequency impedance. It has already been discussed that the total admittance $G_{\mathrm{T}}$ of the probe can be expressed in terms of the sheath admittance $G_{\mathrm{S}}$ and the admittance $G_{\mathrm{P}}$ of the ambient plasma considering that these two admittances are connected in series (OYA, 1965), that gives the relation

$$
G_{\mathrm{T}}=\frac{G_{\mathrm{S}} G_{\mathrm{P}}}{G_{\mathrm{S}}+G_{\mathrm{P}}} .
$$

Numerical calculation gives that in frequency range sufficiently lower than the plasma frequency and the electron cyclotron frequency,

$$
G_{\mathrm{T}} \simeq G_{\mathrm{S}}
$$

since $G_{\mathrm{P}} \gg G_{\mathrm{S}}$ (OYA, 1965). This point was confirmed by the rocket experiment that was carried out in 1966 (Оуа and ОвAYASHI, 1967). The sheath capacitance is characterized by a weak dependence on the frequency of the applied signal as has been seen in the measured impedance value. This constant nature of the admittance $G_{\mathrm{S}}$ of the ion sheath is a reflection of the absence state of the electrons in the ion sheath region; a condenser is therefore formed between the metallic probe surface and the boundary of the ambient plasma, considering the ion sheath region to be vacuum when we use the RF signal which is much higher than the ion cyclotron frequency and ion plasma frequency.

The sheath impedance $Z_{\mathrm{s}}$ measured at the angular frequency $\omega$, for the abrupt sheath model, can be expressed as a capacitance between two concentric spherical shells, that gives a value

$$
Z_{\mathrm{S}}=1 /\left[i \omega 4 \pi \varepsilon_{0}\left(R_{\mathrm{S}} / r_{0}\right)\left(R_{\mathrm{S}}+r_{0}\right)\right]
$$

where $i, R_{\mathrm{S}}$ and $\varepsilon_{0}$ are the unit of the complex quantity, the ion sheath thickness 
and the dielectric constant in vacuum, respectively. The calculation by Aso (1973) at an angular frequency $\omega\left(=0.1 \omega_{\mathrm{P}}\right.$, where $\omega_{\mathrm{P}}$ is the angular plasma frequency) indicates an important result that no significant difference exists between the impedance for the abrupt sheath model and that calculated for the self-consistent sheath structure.

\subsection{Charging effects}

Remarkable charging effects have been deduced from the observed sheath

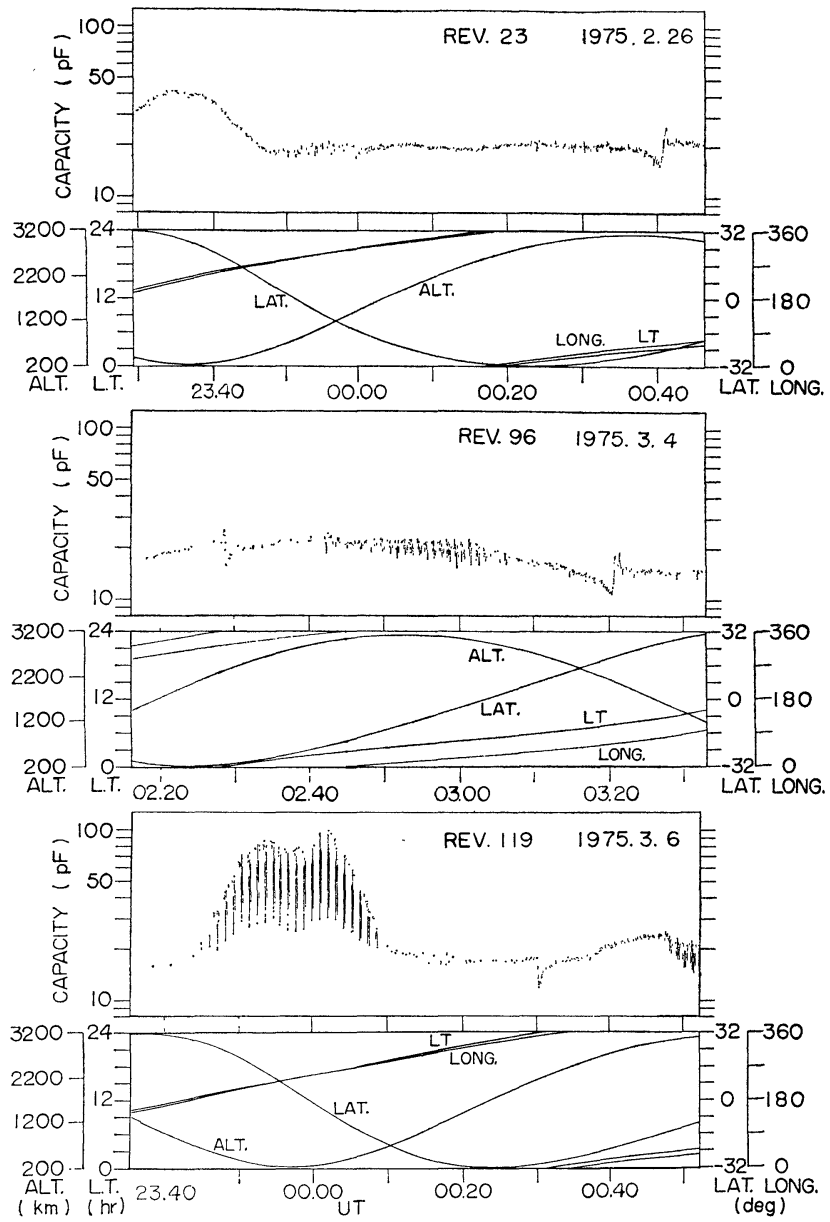

Fig. 9. Examples of the sheath capacity at $406 \mathrm{kHz}$ observed during Revs. 23, 96 and 119; very sharp changes of the sheath capacity are observed in the region of the particle precipitation in the South Atlantic magnetic field anomaly. Sometimes this sharp changes are observable in the region of the magnetic equator. 
capacity of the spherical probe at $406 \mathrm{kHz}$ on board TAIYO satellite (OYA and MorioKA, 1975). In Fig. 9, capacitance data observed at $406 \mathrm{kHz}$ for three example orbits of TAIYO satellite are indicated. The data of the sheath capacitance indicate peculiar variations in the region of the South Atlantic anomaly of the geomagnetic field in almost whole altitude range covered by TAIYO. According to figures the effect obviously extend over a longitude range of at least $180^{\circ}$. This should be compared with results given in the paper of J.A. GLEDHILL (1976; see Ref.). The variation is characterized by plus and minus excursion of the sheath capacitance values centered at average level that is the normal state when the probe is immersed in the ambient plasma. When we make a rough estimation for the normal state assuming $e^{-1}$ hold value for the electron density with respect to the ambient plasma density, the probe potential $\phi$ is approximatively given by

$$
\psi=-(\kappa T / e) .
$$

In the region, where the positive or negative excursion of the observed sheath capacitance value is revealed, the potential can be expressed by using the abrupt sheath model; we can here estimate the potential $\phi$ due to charging up on the probe (OYA and MORIOKA, 1975), as

$$
\phi=-\left(\frac{\kappa T}{e}\right)\left(\frac{R_{\mathrm{P}}}{\lambda_{\mathrm{D}}}\right)^{2}\left\{\frac{x^{2}\left(2 x^{2}+5 x+3\right)}{6(1+x)}\right\}=-\left(\frac{\kappa T}{e}\right)\left(\frac{R_{\mathrm{P}}}{\lambda_{\mathrm{D}}}\right)^{2} \cdot A
$$

where $x=R_{\mathrm{S}} / r_{0}$; and $\lambda_{\mathrm{D}}$ is the Debye's shielding length. When the abrupt sheath model is used $x$ value is given by

$$
x=\frac{6}{C_{\mathrm{S}}-6}
$$

where $C_{\mathrm{S}}$ is the measured sheath capacitance surrounding the sphere probe given in the unit of $\mathrm{pF}$. The measured probe capacity is not indicating a pure spherical component of the capacitance but indicates a parallel state of the spherical and non-spherical components; non-spherical component comes from the near field effect of the boom that is sustaining the spherical probe; i.e., the measured condenser $C_{\text {ob }}$ is given by

$$
C_{\mathrm{ob}}=C_{\mathrm{s}}(N, T)+C_{\mathrm{ns}}(N, T) .
$$

where $C_{\mathrm{s}}(N, T)$ and $C_{\mathrm{ns}}(N, T)$ are the sheath capacitance surrounding the sphere condenser and that surrounding the non-spherical component of the condenser, respectively; both capacities largely depend on the density $N$ and the temperature $T$ of the ambient plasma. Estimation of the non-spherical component $C_{\mathrm{ns}}(N, T)$ have been made to reduce Eq. (8) to Eq. (7) in the limit of normal charging, i.e., 


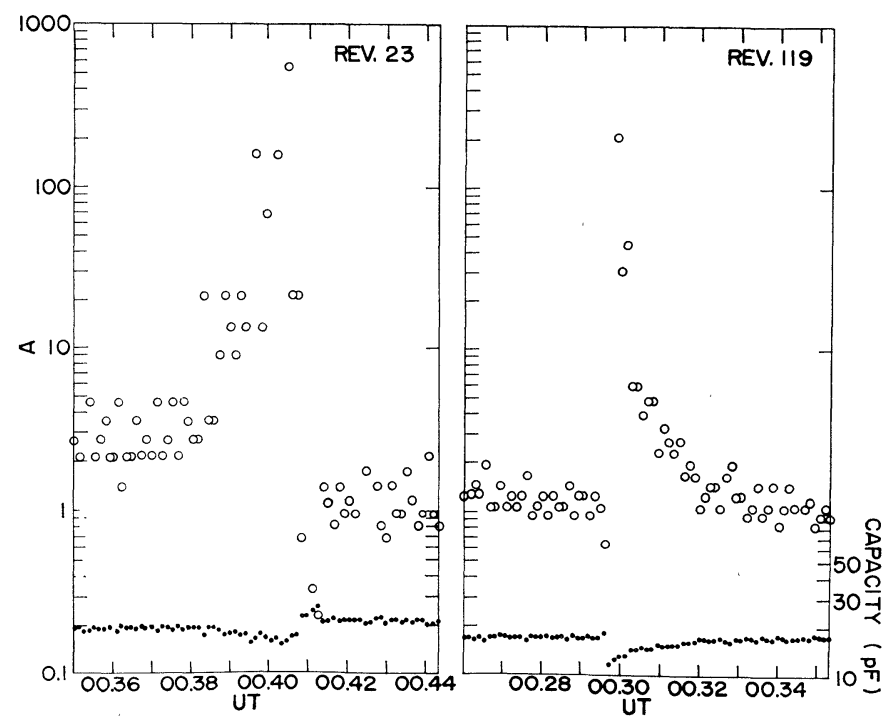

Fig. 10. The calculated potential factor $A$, versus UT around sharp variation of the sheath capacity (given in the bottom of the diagram). The factor $A$ is defined as $\phi=-(\kappa T / e) \cdot\left(R_{\mathrm{P}} / \lambda_{\mathrm{D}}\right) \cdot A$. We can therefore estimate the probe potential $\phi$ compared with the thermal energy of the plasma using $A$ value. The data are the case observed during Rev. 23 and 119.
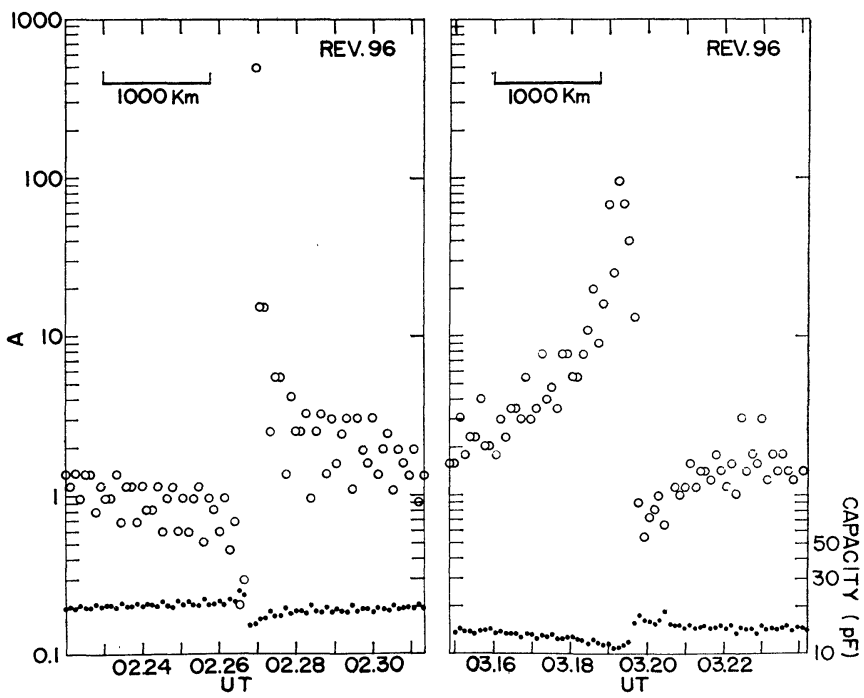

Fig. 11. Same as Fig. 10, obtained during the revolusion 96. 


$$
\left(\frac{R_{\mathrm{P}}}{\lambda_{\mathrm{D}}}\right)^{2}\left\{\frac{x^{2}\left(2 x^{2}+5 x+3\right)}{6(1+x)}\right\}=1 .
$$

Using Eqs. (8) and (11) we can calculate the probe potential due to charging up. In Figs. 10 and 11, the estimated probe potentials have been plotted corresponding to Revs. 23, 96 and 119 . The results indicate that very strong charging effects have been produced; sometimes 30 volts (corresponding to $A=100$, and $T=3,000 \mathrm{~K}$ ) or higher voltage than that is indicated. We need cross check, however, for the final conclusion of this charging up effect by using the instrument that is sensitive to the DC effects. After the passage through the maximum point, the potential quickly changes to small value indicating that the probe potential is almost same with space potential of the ambient plasma. Highly negative potential before the maximum point is caused by the incoming electron flux that exceeds the ion flux, while the shallow potential that is close to space potential results from incoming ion flux that exceeds the electron flux. Since the regions of the electron excess precipitation and ion excess precipitation make a clear separation it is called the differential precipitation (OYA and MORIOKA, 1975).

\section{Artificial Triggering of the Particle Precipitation}

\subsection{Relation to the telemetry radio wave}

The peculiar behavior of the impedance variations can be classified into 5 cases; typical forms of the variation have been given in Fig. 12. The occurrence

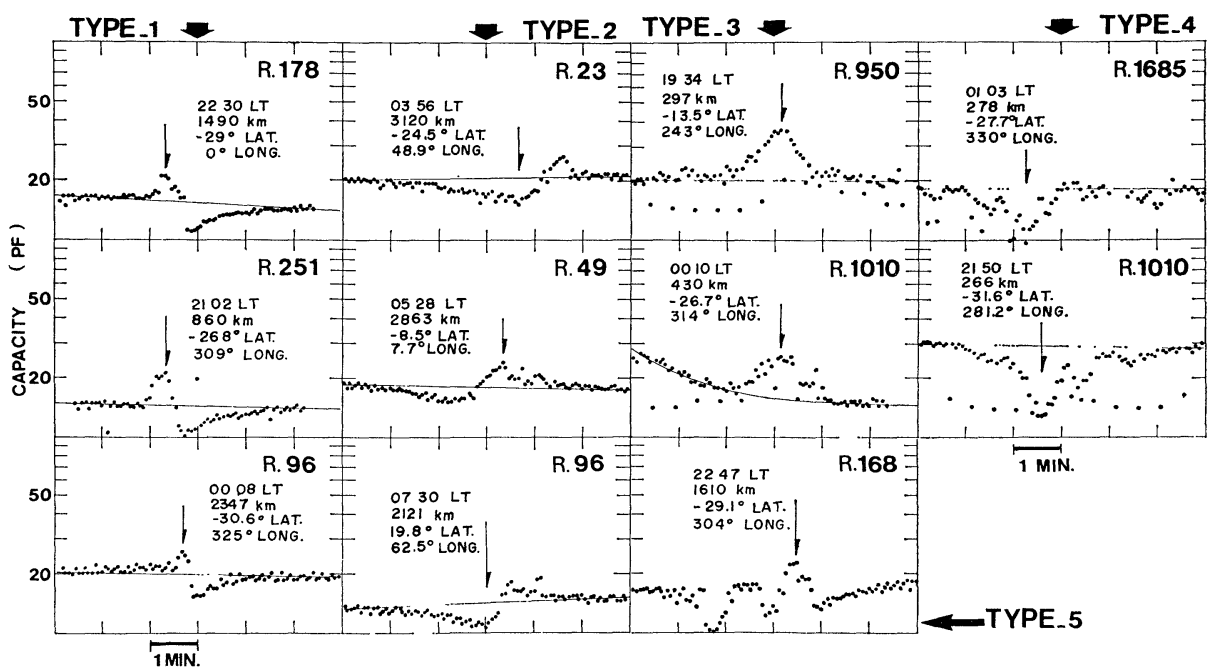

Fig. 12. Classification of the sheath capacitance variation. 


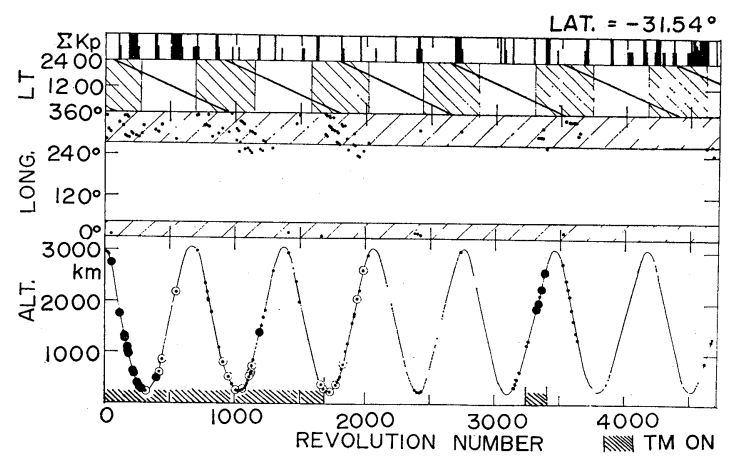

Fig. 13. The record of the occurrence of the sheath capacitance variation with $\Sigma K_{\mathrm{P}}$ and the information on the real time operation of the telemetry radio waves (the period of the real time operation is given by hatched area). The occurrences of the sharp variations of the sheath capacity are plotted on the reference of the satellite orbit; the reference is made as the satellite position at the south end (Lat.= $-31.5^{\circ}$ ) where the region of the South Atlantic anomaly of the magnetic field is covered. Large solid circles indicate type 1 and 2 and open circle with small dots indicate the case no variation has been detected even the observation was made in the region of South Atlantic anomaly.

of these typical pattern of the sheath capacitance variation (or the charging effects) has been summarized in Fig. 13, versus the period after launching. A clear evidence is concluded from these results that only in an initial phase of the TAIYO observation, indication was made of a very sharp variation (type 1 and 2) of the probe impedance. After July 17, 1975 only broad variations of type 3, 4 and 5 of the sheath capacitance can be observed but again type 1 and 2 of the charging effects are revealed during a period from November 21 to December 2. This change at July 17 th make a possible coincidence with stopping of the telemetry radio wave, i.e., after July 17 th, we stopped the real-time transmission of the telemetry radio wave; the observed data were only stored in on-board data recorder. It has then been concluded that the strong charge up effects take place under the condition where the observation is made simultaneously with the real-time transmission of the telemetry radio waves. The interval from November 21th to December 2nd, real-time transmission was again operated. The possible origin of the differential precipitation is, thus, related to the artificial triggering of the precipitation in the region of the South Atlantic magnetic field anomaly.

\subsection{Process of the differential precipitation}

Two basic processes take place surrounding the telemetry antenna which 
is excited at 136 and $400 \mathrm{MHz}$ with the amplitude of several tens volts. One is a ponder-motive force that spatter the electrons outside the area of the strong antenna field and the second is the effect of the electron absorption just on the antenna surface. The spattering of the electron takes place during the negative cycle of the antenna potential while the partial absorption takes place in the positive cycle of the radio wave. Finally, a quasi DC state of the bias potential distribution is produced surrounding the RF antenna whose maximum value easily approaches to the maximum excitation potential (OYA, 1978). The second step of the triggering effect is the transport of the local electric field into the plasma along the magnetic field (see Fig. 14). It is well known that the current can easily flow in the direction parallel to the magnetic field line while it is difficult to flow in the perpendicular direction. This tendency brings a result that the electric potential in the direction parallel to the magnetic field is nearly constant while the potential difference is produced in the direction perpendicular to the magnetic field. As a result, we can expect that the electric field near the satellite can be transported along the magnetic field. There is, then, possibility that the electric field surrounding satellite can penetrate into the region of the particle trapping. A strong $\boldsymbol{E} \times \boldsymbol{B}$ drift takes place giving triggering effects on an unstable region. An important evidence of this triggering effect is that the charging up is possible only when the satellite is located in the region of South Atlantic anomaly. Therefore, we have a conclusion that, an unstable state is required for the effective triggering of the differential precipitation; we should observe the differential precipitation in all regions if the precipitation has hap-

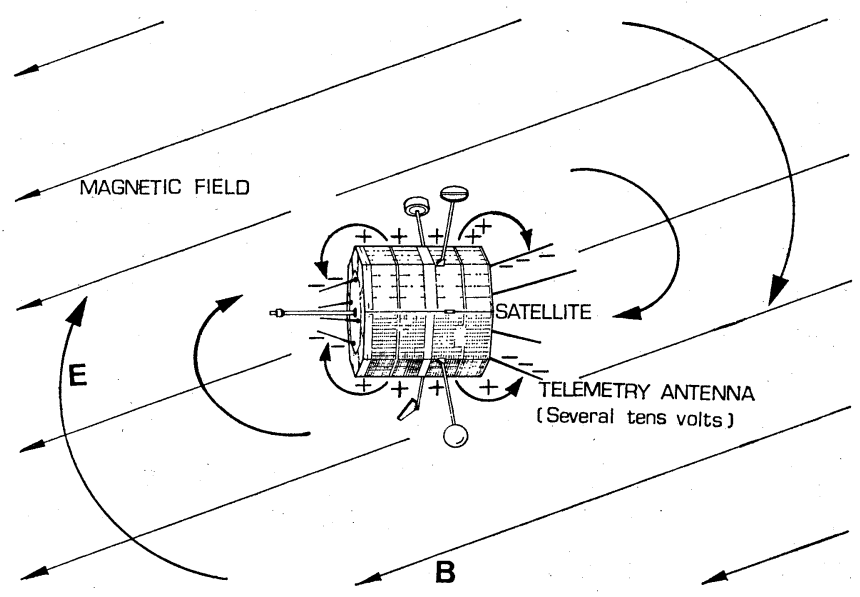

Fig. 14. A schematic illustration of the quasi-DC charging of the satellite and the transmission of the electric field through the ambient plasma. 
pened only as result from triggering effects brought by the artificially produced electric field.

Unstable region is here defined as an area where the particle precipitation is progressing with relatively slow speed. The applied electric field $\boldsymbol{E}$, therefore, only enhances these precipitation phenomena.

\section{Conclusion}

Analyses of the gyro-plasma probe installed on TAIYO satellite have been progressing, to obtain the electron density data at the satellite level and the capacitance value data of the ion sheath region that were measured at $406 \mathrm{kHz}$. Analysed data for 160 orbits provide informations on the various states of the electron density distribution such as local time dependence, quasi-vertical profile, and anomalous distribution of the ionospheric electron density. The local time dependence of the electron density distribution has been checked compared with ISIS data observed in the same period. The result indicates no systematic difference between the ISIS results and TAIYO results.

The sheath capacity has two important informations. The first is the information of the Debye's shielding length from which we can deduce the electron temperature when we have known electron density data or can deduce the electron density when we have informations on the electron temperature.

The second information that can be obtained from the sheath capacitance is the ion sheath thickness for large ion sheath that is produced due to charging up on the probe surface. The charging up effects had frequently happened in a region of the South Atlantic anomaly of the magnetic field associated with the excess precipitation of electrons or the excess precipitation of ions. Usually these two separate regions are connected; the connected situation of the electron excess precipitation and ion excess precipitation regions is called the differential precipitation. It is disclosed that the differential precipitation is triggered by the local electric field surrounding satellite; the most possible origin of this local electric field is the telemetry signal fed to the antenna. A pondermotive force spatter the electron away from the satellite and the strong electric field partially causes absorption of the electrons on the antenna surface. All these effect produces quasi-DC potential surrounding the satellite. The electric field raise the drifting motion of the particle being transmitted in the local region where the particle precipitation is dominated as the case of the South Atlantic anomaly of the geomagnetic field. The precipitation effects are thus enhanced by the transmission of the local electric fields. These evidence gives the important suggestion that we should be careful to make measurement of the low energetic component of the particle in a region where the particles are pre- 
cipitating as in the region of South Atlantic anomaly of the geomagnetic field. This may also be important in the auroral region.

The authors would like to express their thanks to Prof. K. Hirao for his arrangement of TAIYO satellite observation program as the manager of the team and also would like to express thanks to the observation team of Institute for Space and Aeronautical Science, University of Tokyo. The IMP data aquisition had been carried out by IMP observation team, the authors are largely indebted to Mr. M. Kondo for his observation works. We are grateful to Dr. I. Vette for ISIS-2 data provided by WDC center at NASA.

\section{REFERENCES}

Aso, T., Impedance of an ion-sheathed spherical probe in a warm, isotropic plasma, Radio Sci., 8, 139-146, 1973.

BUCKLeY, R., A theory of resonance rectification: The response of a spherical plasma probe to alternating potentials, Proc. Roy. Soc., Ser. A, 290, 186-219, 1966.

BUCKLEY, R., The response of a spherical plasma probe to alternating potentials: Results of computations and use for plasma diagnostics, J. Plasma Phys., 1, 171-191, 1967.

Ejiri, M., H. Oya, T. Aso, C. Morita, T. Obayashi, S. Urimoto, and H. Yamaki, The gyroplasma probe on-board the REXS-DENPA satellite, Inst. Space and Aeronautical Sci., Univ. of Tokyo, Report No. 495, 1973.

Gledhill, J.A., Aeronomic effects of the South Atlantic anomaly, Rev. Geophys. Space Phys., 14, 173-187, 1976.

KIsT, R., Ion sheath effects on RF plasma probe: Experimental results in laboratory plasmas, Radio Sci., 6, 921-934, 1977.

OYA, H., Effect of resonances on the admittance of an RF plasma probe surrounded by an ion sheath, Rep. Ionos. Space Res. Jpn., 19, 243-271, 1965.

Oya, H. and T. OBayashi, Measurement of ionospheric electron density by a gyro-plasma probe: A rocket experiment by a new impedance probe, Rep. Ionos. Space Res. Japan, 20, 199-213, 1966.

Oya, H. and T. OвауASHI, Rocket measurement of the ionospheric plasma by gyro-plama probe, Rep. Ionos. Space Res. Jpn., 21, 1-8, 1967.

OyA, H. and A. MORIOKA, Instrumentation and observation of gyro-plasma probe installed on TAIYO for measurement of ionospheric plasma parameters and low energetic particle effects, J. Geomag. Geoelectr., 27, 331-361, 1975.

OyA, H., Generation mechanism of proton cyclotron echoes due to pulsed radio frequency waves in space plasma, J. Geophys. Res., 83, 1991-2008, 1978. 